\title{
Alongamento Muscular Segmentar Melhora Função e Alinhamento do Joelho de Indivíduos com Síndrome Femoropatelar: Estudo Preliminar
}

\section{Segmental Muscular Stretching Improves Knee Function and Alignment in Subjects With Patellofemoral Syndrome: Preliminary Study}

Gisela Cristiane Miyamoto Fernanda Regina Soriano

Cristina Maria Nunes Cabral

Universidade Cidade de São Paulo (Unicid) - São Paulo, SP

Endereço para correspondência: Cristina Maria Nunes Cabral Programa de Mestrado em Fisioterapia, Universidade Cidade de São Paulo

Rua Cesário Galeno, 475, Tatuapé 03071-000 -São Paulo, SP

E-mail: ccabral@cidadesp.edu.br

\section{RESUMO}

Introdução: A síndrome femoropatelar (SFP) é uma desordem dolorosa comum do joelho e para seu tratamento normalmente são utilizados exercícios de fortalecimento do músculo quadríceps femoral, sendo que poucos trabalhos encontrados na literatura investigaram os efeitos diretos do alongamento muscular. Objetivo: O objetivo deste estudo foi avaliar os efeitos do alongamento muscular segmentar no tratamento de pacientes com SFP. Métodos: Participaram do estudo 12 voluntários com SFP, dominância de membro inferior direito e idade média de 20 anos. As variáveis funcionais avaliadas antes e depois do tratamento foram: ângulo Q, intensidade da dor, capacidade funcional pela escala de contagem de Lysholm, sensação de posição articular (SPA) a 40 e 50 graus de flexão do joelho, trabalho total e momento de força concêntrico dos músculos quadríceps femoral e isquiotibiais a 60 e 180\%/s. Após a avaliação inicial, foi realizado o tratamento que consistiu em alongamento muscular segmentar bilateral dos músculos isquiotibiais, tríceps sural e quadríceps femoral, com duração de 30 segundos e 10 repetições para cada músculo. As variáveis avaliadas antes e após o tratamento foram analisadas pelo teste $t$ para amostras dependentes $(a<0,05)$. Resultados: Todas as variáveis apresentaram diferença significante após o tratamento $(p<0,05)$, exceto $o$ momento de força e trabalho total dos músculos extensores a $60 \%$, trabalho total dos extensores e flexores a 180\%/s e a SPA a 50 e 40 graus de flexão do joelho. Conclusão: Os resultados desta pesquisa permitem inferir que o tratamento com alongamento muscular segmentar possibilita melhoras de vários sinais e sintomas apresentados pelos pacientes com SFP, como alinhamento, dor e função do joelho.

Palavras-chave: exercícios de alongamento muscular/métodos, síndrome da dor patelofemoral, terapia por exercício.

\section{ABSTRACT}

Introduction: Patellofemoral syndrome (PFS) is a common painful knee disorder and for its treatment, quadriceps femoris strengthening exercises are normally used; however, few studies in the literature investigate the direct effects of stretching exercises. Objective: In order to fill this gap, the objective of this study was to evaluate the effects of segmental stretching exercises on the treatment of patients with PFS. Methods: Twelve PFS patients with right foot dominance and mean age of 20 years were evaluated. The following functional variables were assessed, before and after treatment: $Q$ angle, pain intensity, knee functional injury level (Lysholm scale), joint position sense (JPS) at 40 and 50 degrees of knee flexion and total work and concentric torque of quadriceps femoris and hamstring muscles at 60 and 180 degrees/sec. After initial evaluation, bilateral segmental stretching exercises for hamstring, triceps surae and quadriceps femoris muscles were performed, with 30-second duration and 10 repetitions on each muscle. Data obtained before and after treatment were analyzed by $t$ test for dependent samples $(a<0.05)$. Results: All variables showed significant difference after treatment $(p<0.05)$, except extensor muscular torque and total work at 60 degrees/sec, extensor and flexor total work at 180 degrees/sec and JPS at 40 and 50 degrees of knee flexion. Conclusion: Based on these results, we can state that segmental stretching exercises treatment improves major PFS signals and symptoms of patients, such as alignment, pain and knee function.

Keywords: muscle stretching exercises/methods, patellofemoral pain syndrome, exercise therapy. 


\section{INTRODUÇÃO}

A síndrome femoropatelar (SFP) é uma das desordens dolorosas mais comuns que acometem o joelho, compreendendo cerca de 25\% das lesões de joelho tratadas em clínicas de medicina esportiva(1). De acordo com Thomeé et al. ${ }^{(2)}$, os problemas no joelho representam entre 23 a 31\% das lesões ou queixas nessas clínicas, sendo as condições dolorosas relacionadas à articulação femoropatelar as mais comuns. Tem início insidioso, caracterizado por dor anterior ou retropatelar durante atividades funcionais na ausência de outras lesões musculoesqueléticas ${ }^{(3,4)}$. A etiologia ainda é indefinida e multifatorial, caracterizada principalmente pelo desequilíbrio entre os componentes mediais e laterais do músculo quadríceps femoral|(5). Este desequilíbrio desencadeia mau alinhamento patelar, dor e, consequentemente, diminuição da propriocepção(6). A alteração da sensação de posição articular (SPA) pode também estar relacionada com a diminuição da flexibilidade, pois o encurtamento muscular promove um mau alinhamento patelar desencadeando dor ${ }^{(7)}$. Porém, ainda não há estudos na literatura que comprovem a eficácia do alongamento muscular na melhora da propriocepção. Apenas o estudo de Larsen et al. ${ }^{(8)}$ avaliou indivíduos saudáveis após alongamento estático dos músculos quadríceps femoral e isquiotibiais, não observando modificação da SPA.

A avaliação isocinética é importante instrumento para verificar o pico do momento de força do músculo quadríceps femoral destes pacientes ${ }^{(9)}$. Hazneci et al. ${ }^{(6)}$ observaram que pacientes com SFP apresentam momento de força e trabalho total dos músculos quadríceps femoral e isquiotibiais a 60 e 180\%/s e SPA diminuídos em relação aos indivíduos saudáveis. No entanto, após a realização de exercícios isocinéticos, demonstraram melhora da SPA em pacientes com SFP(6). Outros trabalhos sugerem existir uma relação direta entre a dor e a capacidade funcional dos pacientes com SFP, o que demonstra a importância da avaliação da intensidade da dor, que pode causar limitações ao paciente ${ }^{(3,10)}$. Sacco et al. ${ }^{(10)}$ observaram melhora da capacidade funcional sem redução concomitante da dor após um protocolo de intervenção baseado em fortalecimento e alongamento muscular em pacientes com SFP.

Visto que o alongamento muscular pode promover redução da intensidade dos sinais e sintomas apresentados por pacientes com SFP, este estudo teve como objetivos avaliar o ângulo Q, intensidade da dor, capacidade funcional, SPA do joelho em dois diferentes ângulos alvo e momento de força e trabalho total dos músculos flexores e extensores do joelho antes e após um período de alongamento muscular segmentar.

\section{MÉTODOS}

\section{Sujeitos}

Participaram deste estudo 13 indivíduos sedentários (sem prática de atividade física regular) com SFP de ambos os gêneros, com idade entre 18 e 30 anos. Todos os pacientes assinaram um termo de consentimento livre e esclarecido e os procedimentos da pesquisa foram realizados após aprovação do Comitê de Ética em Pesquisa Institucional, sob protocolo no 13.316 .657$.

Para que o indivíduo fosse classificado como portador de SFP, foram considerados os seguintes critérios de inclusão: presença de três sinais ou sintomas clínicos observados na avaliação física (ângulo Q maior que 14 graus para homens e 17 graus para mulheres, mau alinhamento patelar, dor à palpação das facetas articulares, pronação subtalar excessiva e teste de compressão patelar positivo); relato de dor anterior ou retropatelar do joelho há, pelo menos, seis meses, durante ou após atividades de agachar, subir ou descer escadas, permanecer por um tempo prolongado na posição sentada, ajoelhar, correr e saltar; início insidioso dos sintomas sem relação com evento traumático; e encurtamento dos músculos isquiotibiais considerando uma perda de pelo menos 30 graus para a extensão total do joelho(11-13). Os critérios de exclusão foram: apresentar sinais e sintomas de qualquer outra lesão musculoesquelética em membros inferiores; escore da escala de contagem de Lysholm maior que 90; e três faltas seguidas sem reposição(13). Um voluntário foi excluído do estudo devido às faltas, de forma que permaneceram 12 voluntários no período de alongamento.

O tamanho da amostra foi calculado usando $85 \%$ de poder estatístico para detectar uma diferença de $25 \%$ após o tratamento. O N $\leq 5 \%$ e intervalo de confiança de $95 \%$ foram considerados significantes.

\section{AVALIAÇÃO}

Primeiramente os voluntários foram submetidos a uma avaliação física, feita por fisioterapeuta, para confirmação dos critérios de inclusão, por meio da realização de testes especiais e funcionais do joelho e da articulação femoropatelar e coleta dos dados antropométricos. As variáveis foram avaliadas antes e após tratamento fisioterapêutico. Quando a sintomatologia era bilateral, foi escolhido o membro inferior mais acometido, ou seja, com maior número de sinais e sintomas, para avaliação.

O ângulo Q foi mensurado com goniômetro, estando o indivíduo em decúbito dorsal, pés perpendiculares à maca e músculo quadríceps femoral relaxado(1). A intensidade da dor no joelho foi avaliada pela escala visual analógica - EVA ${ }^{(5)}$ e a capacidade funcional do joelho pela escala de contagem de Lysholm ${ }^{(14)}$.

A SPA do joelho e o momento de força e trabalho total dos músculos quadríceps femoral e isquiotibiais foram mensurados no dinamômetro isocinético Cybex Norm (CSMI Solutions, 101 Tosca Drive, Stoughton, MA, 02072, EUA). Antes do teste foi realizado um aquecimento prévio de cinco minutos na bicicleta estacionária, seguido de alongamentos de músculos dos membros inferiores. O indivíduo foi posicionado na cadeira do dinamômetro na posição sentada com estabilização de cintos na região do tronco, abdome e coxa, a fossa poplítea posicionada pelo menos três centímetros fora do assento da cadeira, com o tronco, quadril e joelhos fletidos a 90 graus e o eixo articular alinhado com o eixo do dinamômetro, previamente calibrado de acordo com os procedimentos técnicos descritos no manual do fabricante. Para a avaliação da SPA, os olhos foram vendados. $O$ teste iniciou em dois diferentes ângulos de flexão do joelho, que foram selecionados de forma aleatória. Na primeira etapa, o joelho do paciente foi passivamente estendido até o ângulo alvo de 50 graus, partindo de 90, e a posição foi mantida por 10 segundos. Após esse período, o examinador moveu o joelho novamente para a posição de 90 graus de flexão. Na segunda etapa, o joelho do paciente foi passivamente fletido até o ângulo alvo de 40 graus, partindo de zero. Em cada etapa foi pedido para que o paciente reproduzisse ativamente a posição articular por três vezes, avisando o examinador quando a posição fosse atingida, o que foi considerado como ângulo estimado ${ }^{(6)}$.

Para a avaliação do momento de força, foi retirada a venda dos olhos e o indivíduo permaneceu no mesmo posicionamento, porém com os membros superiores cruzados no tórax para evitar compensações. O momento de força e trabalho total foram mensurados durante os movimentos de flexão e extensão do joelho na amplitude de zero a 90 graus de forma concêntrica nas velocidades angulares de 60 e 180 \% . Foi realizado aquecimento prévio com três repetições para cada velocidade angular, para aprendizado do movimento. Na avaliação foram realizadas cinco repetições para cada velocidade angular estipulada, em que o voluntário era estimulado durante toda execução dos movimentos com comando verbal: "Força, força, força"(3,6).

\section{Tratamento}

Após a avaliação inicial, todos os voluntários realizaram o tratamento três vezes por semana, durante seis semanas. O tratamento consistiu em autoalongamento segmentar dos músculos isquiotibiais (paciente em decúbito dorsal com quadril flexionado e joelho estendido), quadríceps femoral (paciente em posição ortostática com hiperextensão 
do quadril e flexão do joelho) e tríceps sural (paciente em posição ortostática com quadril e joelho estendidos e tornozelo em dorsiflexão) bilateralmente mantendo por 30 segundos e repousando pelo mesmo tempo com 10 repetições cada músculo. Os procedimentos de avaliação e reavaliação e o de tratamento foram realizados sempre pelo mesmo pesquisador, de forma que o que realizou o tratamento não teve acesso aos dados de avaliação dos pacientes.

\section{Análise estatística}

Toda a análise estatística foi realizada com 5\% de significância. Inicialmente, a normalidade dos dados foi analisada pelo teste de ShapiroWilk e a homogeneidade de variância pelo teste de Levene. Como todas as variáveis apresentaram distribuição normal, foram analisadas antes e após o tratamento pelo teste $t$ para amostras dependentes.

\section{RESULTADOS}

Os dados demográficos da população estudada demonstraram média de idade de $20 \pm 2$ anos, massa de 68,68 $\pm 14,29 \mathrm{~kg}$, estatura de $1,7 \pm 0,1 \mathrm{~m}$ e IMC de $24,6 \pm 3,6 \mathrm{~kg} / \mathrm{m}^{2}$. Além disso, dos 12 sujeitos avaliados, quatro eram do gênero masculino e oito, do feminino, sendo o acometimento maior no joelho direito (58,3\%) e todos apresentavam dominância em membro inferior direito.

Após o tratamento, houve diferença estatisticamente significante no ângulo $Q(p=0,003)$ e capacidade funcional ( $p=0,002)$ (figura 1 ). A intensidade da dor também apresentou diferença significante $(p=$ 0,018 ), sendo que as médias dos valores antes do tratamento foram 1,6 $\pm 1,9 \mathrm{~cm}$ e após $0,2 \pm 0,3 \mathrm{~cm}$. Por outro lado, não foi observada diferença significante na SPA a 50 graus de flexão $(p=0,558)$ e SPA a 40 graus de flexão ( $p=0,810$ ) (figura 2).

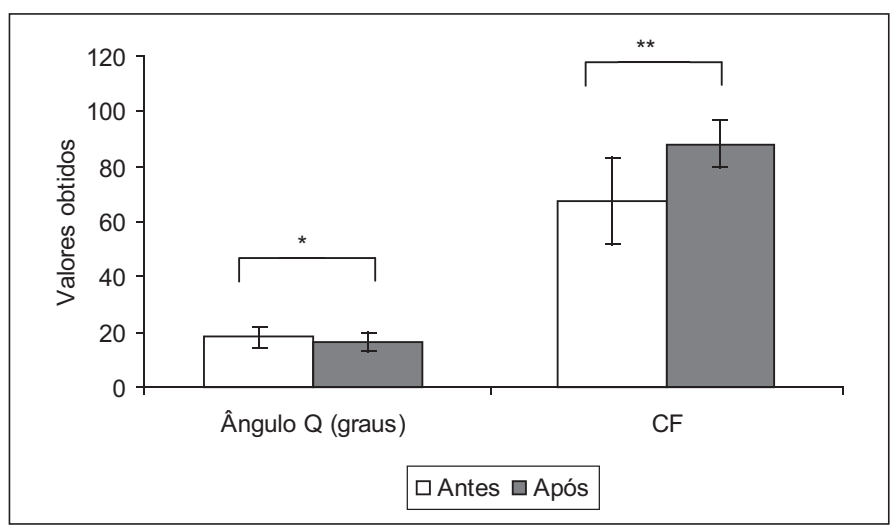

* Diferença estatisticamente significante após o tratamento $(p=0,003)$

** Diferença estatisticamente significante após o tratamento $(p=0,002)$

(CF: capacidade funcional)

Figura 1. Comparação dos valores médios de ângulo Q (graus) e capacidade funcional antes e após o tratamento $(n=12)$.

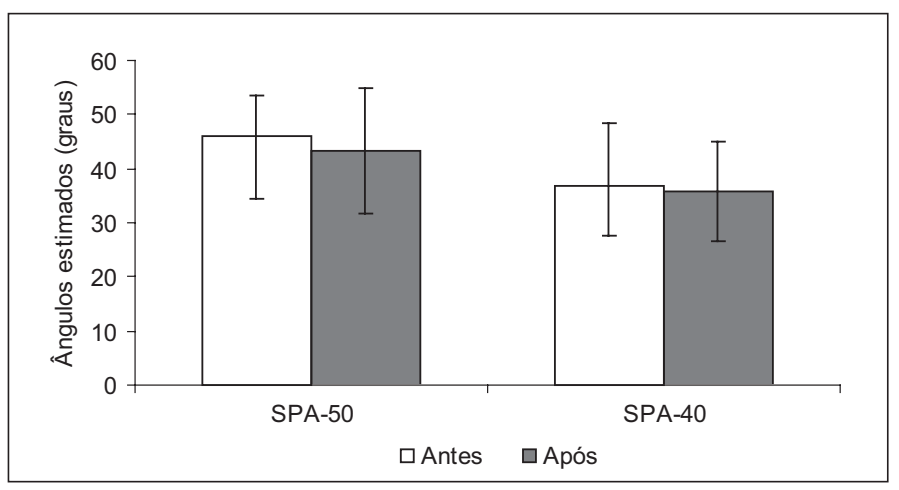

(SPA-50: sensação de posição articular a 50 graus de flexão do joelho;

SPA-40: sensação de posição articular a 40 graus de flexão do joelho)

Figura 2. Valores médios dos ângulos estimados de sensação de posição articular (graus) a 50 e 40 graus de flexão do joelho antes e após o tratamento ( $n=12)$.
Em relação ao desempenho isocinético dos pacientes, foi observada diferença estatisticamente significante no momento de força dos músculos extensores a 180\%/s $(p<0,001)$, dos músculos flexores a $180 \%$ s $(p=0,027)$ e dos músculos flexores a $60 \%$ s $(p=0,008)$, enquanto não houve diferença significante no momento de força dos músculos extensores a $60 \%$ s $(p=0,507)$ (tabela 1). Para a variável trabalho total, houve diferença estatisticamente significante apenas para o dos músculos flexores a $60 \%$ s $(p=0,037)$, sendo que os outros músculos não mostraram diferença significante após o tratamento (músculos extensores a 180\%/s: $p=0,379$; músculos flexores a 180\%/s: $p=0,290$; músculos extensores a 60\%/s: $p=0,591$ ) (tabela 2).

Tabela 1. Médias (desvio padrão) do momento de força dos músculos extensores e flexores do joelho antes e após o tratamento e valores de $p(n=12)$.

\begin{tabular}{c|c|c|c}
\hline Momento de força & Antes & Após & p \\
\hline Músculos extensores a 180\%/s (Nm) & $82,8(30,3)$ & $98(32,9)$ & $<0,001^{*}$ \\
\hline Músculos flexores a 180\%/s (Nm) & $44,8(28,4)$ & $59,08(33,9)$ & $0,027^{*}$ \\
\hline Músculos extensores a 60\%/s (Nm) & $142,9(50,7)$ & $146,83(51,2)$ & 0,507 \\
\hline Músculos flexores a 60\%/s (Nm) & $67,1(36,7)$ & $85,25(41,9)$ & $0,008^{*}$ \\
\hline
\end{tabular}

* Diferença estatisticamente significante antes e após o alongamento $(p<0,05)$.

Tabela 2. Médias (desvio padrão) do trabalho total dos músculos extensores e flexores do joelho antes e após o tratamento e valores de $p(n=12)$.

\begin{tabular}{c|c|c|c}
\hline Trabalho total & Antes & Após & p \\
\hline Músculos extensores a 180\%/s $(J)$ & $93,6(38,9)$ & $98,3(32,9)$ & 0,379 \\
\hline Músculos flexores a 180\%/s $(J)$ & $47,8(37,2)$ & $56,8(36,9)$ & 0,290 \\
\hline Músculos extensores a 60\%/s $(J)$ & $137,6(50,2)$ & $133,3(49,3)$ & 0,591 \\
\hline Músculos flexores a 60\% $(J)$ & $65,6(37,9)$ & $77,4(41,3)$ & $0,037^{*}$ \\
\hline
\end{tabular}

\section{DISCUSSÃO}

A partir dos resultados apresentados, observou-se que o ângulo Q apresentou melhora significante após alongamento muscular segmentar. No trabalho realizado por Tunay et al. ${ }^{(15)}$, foi demonstrada redução significante do ângulo Q em todos os pacientes após diferentes tipos de tratamento. Porém, os autores não descrevem detalhadamente os exercícios realizados, tornando difícil explicar os motivos do realinhamento patelar. Resultado similar foi encontrado neste estudo após a realização de alongamento muscular, em que ocorreu melhora do alinhamento patelar provavelmente devido à redução do encurtamento muscular, que consequentemente diminuiu o estresse articular e a dor. Diferentes resultados foram encontrados por Sacco et al.(10), que não constataram diferença no ângulo Q em indivíduos com SFP após intervenção com alongamento e fortalecimento muscular de cinco semanas, sendo que os autores observaram valores muito baixos do ângulo $Q$, talvez por diferentes mensurações das adotadas neste estudo.

Neste estudo houve redução da intensidade da dor, que apresentou melhora significante após o alongamento muscular segmentar decorrente da melhora do alinhamento articular. Este resultado é semelhante ao de Alaca et al.(3) que observaram redução da intensidade da dor após exercícios isocinéticos, decorrente de aumento da força muscular, com melhora do equilíbrio dinâmico mantendo as forças adequadas sobre a patela e contribuindo para a melhora do alinhamento patelar. Christou ${ }^{(16)}$, após intervenção com taping patelar, também observou redução da dor, devido a maior ativação do músculo vasto medial, promovendo realinhamento patelar. Cabral et al. ${ }^{(17)}$, após alongamento 
muscular segmentar e reeducação postural global, obtiveram redução da intensidade da dor avaliada pela EVA decorrente da melhora da flexibilidade e também do alinhamento patelar. Tyler et al. ${ }^{(13)}$ obtiveram resultados semelhantes quanto à diminuição da intensidade de dor com intervenções visando o fortalecimento dos músculos flexores, extensores, abdutores e adutores de quadril e alongamento dos músculos flexores de quadril e do trato iliotibial, já que os músculos do quadril têm relação direta com o mau alinhamento patelar devido à alteração do posicionamento da pelve. Porém, discussão mais aprofundada entre os trabalhos fica prejudicada, devido às diferentes abordagens terapêuticas utilizadas pelos autores.

A presença de dor afeta a realização de atividades funcionais dos pacientes com SFP(3,18). Desta forma, a efetividade dos tratamentos pode estar relacionada com a diminuição da dor, o que foi obtido neste estudo após a realização do alongamento dos três grupos musculares, já que esse sintoma promove limitações funcionais. Além disso, observou-se também melhora significante da capacidade funcional em todos os voluntários, o que sugere melhora da sintomatologia dos pacientes na realização de suas atividades. Resultados semelhantes foram encontrados por Alaca et al. ${ }^{(3)}$, que realizaram exercícios isocinéticos para fortalecimento e alongamento muscular de membros inferiores e também avaliaram a capacidade funcional pela escala de contagem de Lysholm. Peeler e Anderson ${ }^{(7)}$ obtiveram melhora da intensidade da dor e da capacidade funcional em pacientes com SFP após três semanas de alongamento muscular segmentar do músculo quadríceps femoral, porém utilizaram formas de avaliação dessas variáveis diferentes das adotadas no presente estudo.

O momento de força dos músculos extensores e flexores a 180\%/ e o momento de força e trabalho total dos músculos flexores a $60 \%$ s apresentaram melhora significante após alongamento muscular segmentar. Para Anderson e Herrington ${ }^{(19)}$ o desempenho do momento de força concêntrico e excêntrico do músculo quadríceps femoral em velocidade angular de $30 \%$ s de indivíduos com SFP está diminuído. Thomeé(5) afirmou que a dor durante a realização de atividades presente na SFP leva a diminuição da atividade física e consequente diminuição na produção de força do músculo quadríceps femoral. Alaca et al. ${ }^{(3)}$ e Hazneci et al. ${ }^{(6)}$ obtiveram aumento do pico do momento de força e trabalho total dos músculos quadríceps femoral e isquiotibiais com velocidade angular de 60 e 180\%/s após fortalecimento muscular com exercícios isocinéticos. No entanto, este estudo não observou aumento significativo no pico do momento de força e no trabalho

\section{REFERÊNCIAS}

1. Fredericson M, Yoon K. Physical examination and patellofemoral pain syndrome. Am J Phys Med Rehabil 2006;85:234-43.

2. Thomeé R, Augustsson J, Karlsson J. Patellofemoral pain syndrome: a review of current issues. Sports Med 1999;28:245-62.

3. Alaca R, Yilmaz B, Goktepe AS, Mohur H, Kalyon TA. Efficacy of isokinetic on functional capacity and pain in patellofemoral syndrome. Am J Phys Med Rehabil 2002;81:807-13.

4. Nijs J, Geel CV, Auwera CV, Velde BV. Diagnostic value of five clinical tests in patellofemoral pain syndrome. Man Ther 2006;11:69-77.

5. Thomeé, R. A comprehensive treatment approach for patellofemoral pain syndrome in young women. Phys Ther 1997;77:1690-703.

6. Hazneci B, Yildiz Y, Sekir U, Aydin T, Kalyon TA. Efficacy of isokinetic exercise on joint position sense and muscle strength in patellofemoral pain syndrome. Am J Phys Med Rehabil 2005;84:521-7.

7. Peeler J, Anderson JE. Effectiveness of static quadriceps stretching in individuals with patellofemoral joint pain. Clin J Sport Med 2007;17:234-41.

8. Larsen R, Lund H, Christensen R, Rogind H, Danneskiold-Samsoe B, Bliddal H. Effect of static stretching of quadriceps and hamstring muscles on knee joint position sense. Br J Sports Med 2005;39:43-6.

9. Thomeé R, Grimby G, Svantesson U, Österberg U. Quadriceps muscle performance in sitting and standing in young women with patellofemoral pain syndrome and young healthy women. Scand Med Sci Sports 1996;6:233-41

10. Sacco ICN, Konno GK, Rojas GB, Arnone AC, Pássaro AC, Marques AP, et al. Functional and EMG responses to a physical therapy treatment in patellofemoral syndrome patients. J Electromyogr Kinesiol 2006;16:167-74. total dos músculos extensores em velocidade angular de $60 \%$, apresentou somente diferença significante no valor dos músculos flexores a $60 \%$ s após o tratamento, mostrando melhora da força muscular desses músculos.

A alteração da SPA de pacientes com SFP pode ser explicada pela presença de dor e pelo aumento dos estresses articulares em consequência de mau alinhamento patelar ${ }^{(6,20,21)}$. Dentre os achados deste estudo, todos os pacientes apresentaram alteração da SPA, porém não houve diferença significante da SPA com $50^{\circ}$ e $40^{\circ}$ de flexão após o tratamento. Os resultados da SPA deste estudo podem ter sofrido influência da presença de dor durante a avaliação, já que a dor poderia ser um feedback para a identificação do posicionamento articular e como ao final do tratamento todos os pacientes obtiveram diminuição significante da intensidade da dor, estes teriam perdido este input sensorial. Dados semelhantes foram encontrados no estudo de Larsen et al. ${ }^{(8)} \mathrm{em}$ que não foi observada melhora significante da SPA após alongamento muscular de indivíduos saudáveis. No entanto, Hazneci et al. ${ }^{(6)}$ observaram melhora da SPA após exercício isocinético, enquanto Lobato et al. ${ }^{(21)}$ verificaram que não houve diferença significante na propriocepção dos joelhos dos indivíduos com SFP, quando comparados com indivíduos sem lesão musculoesquelética. Assim, torna-se evidente que a avaliação da SPA após a realização de exercícios deve ser largamente realizada, para a obtenção de resultados mais homogêneos.

Além disso, sugere-se que outros estudos sobre os efeitos do alongamento muscular no tratamento da SFP sejam realizados, avaliando atletas de diferentes modalidades, já que a escolha de indivíduos sedentários, mesmo que tomada para garantir maior homogeneidade da amostra, limitou o número de indivíduos incluídos neste estudo.

\section{CONCLUSÃO}

Pode-se inferir que o alongamento muscular segmentar dos músculos isquiotibiais, quadríceps femoral e tríceps sural possibilitam meIhoras importantes nos principais sinais e sintomas apresentados pelos pacientes com SFP. Desta forma, sugere-se que estes exercícios de alongamento muscular também devem ser indicados para o tratamento destes indivíduos. Porém, existem evidências de que essa técnica não melhore a propriocepção de pacientes.

Todos os autores declararam não haver qualquer potencial conflito de interesses referente a este artigo.
11. Bandy WD, Irion JM, Briggler M. The effect of time and frequency of static stretching on flexibility of the hamstring muscles. Phys Ther 1997;77:1090-6.

12. Magee DJ. Joelho. In: Magee DJ, editor. Avaliação musculoesquelética. São Paulo: Manole 2002;525-619

13. Tyler TF, Nicholas SJ, Mullaney MJ, McHugh MP. The role of hip muscle function in the treatment of patellofemoral pain syndrome. Am J Sports Med 2006;34:630-6.

14. Peccin MS, Ciconelli R, Cohen M. Questionário específico para sintomas do joelho "Lysholm knee scoring scale" - tradução e validação para a língua portuguesa. Acta Ortop Bras 2006;14:268-72.

15. Tunay VB, Baltaci G, Tunay S, Ergun N. A comparison of different treatment approaches to patellofemoral pain syndrome. The Pain Clinic 2003;15:179-84.

16. Christou EA. Patellar taping increases vastus medialis oblique activity in the presence of patellofemoral pain. J Electromyogr Kinesiol 2004;14:495-504.

17. Cabral CMN, Yumi C, Sacco ICN, Casarotto RA, Marques AP. Eficácia de duas técnicas de alongamento muscular no tratamento da síndrome femoropatelar: um estudo comparativo. Fisioterapia e Pesquisa 2007;14:48-56.

18. Tunay VB, Ergun N, Baltaci G, Tunay S, Erden Z. Treatment of patellar tracking and pain in patellofemoral malalignment: conservative versus surgery. The Pain Clinic 2003;15:185-92.

19. Anderson G, Herrington L. A comparison of eccentric isokinetic torque production and velocity of knee flexion angle during step down in patellofemoral pain syndrome patients and unaffected subjects. Clin Biomech 2003;18:500-4.

20. Baker V, Bennell K, Stillman B, Cowan S, Crossley K. Abnormal knee joint position sense in individuals with patellofemoral pain syndrome. J Orthop Res 2002;20:208-14.

21. Lobato DFM, Santos GM, Coqueiro KRR, Mattiello-Rosa SMG, Terruggi-Junior A, Bevilaqua-Grossi D, et al. Avaliação da propriocepção do joelho em indivíduos portadores de disfunção femoropatelar. Rev Bras Fisioter 2005;9:57-62. 